\title{
Determination of Important Parameters in Affecting the Uptake of Reactive Black 5 by Chitosan Beads through Statistical Approach
}

\author{
Yi-Pin Phung, ${ }^{1}$ Siew-Teng Ong, ${ }^{1}$ and Pei-Sin Keng ${ }^{2}$ \\ ${ }^{1}$ Department of Chemical Science, Faculty of Science, Universiti Tunku Abdul Rahman, Jalan Universiti, Bandar Barat, \\ 31900 Kampar, Perak, Malaysia \\ ${ }^{2}$ Department of Pharmaceutical Chemistry, International Medical University, No.126, Jalan Jalil Perkasa 19, Bukit Jalil, \\ 57000 Kuala Lumpur, Malaysia
}

Correspondence should be addressed to Siew-Teng Ong; ongst_utar@yahoo.com

Received 20 May 2013; Revised 10 July 2013; Accepted 14 July 2013

Academic Editor: Hani El-Nezami

Copyright (c) 2013 Yi-Pin Phung et al. This is an open access article distributed under the Creative Commons Attribution License, which permits unrestricted use, distribution, and reproduction in any medium, provided the original work is properly cited.

Chitosan which can be obtained from fishery waste was studied as an alternative source to remove pollutants in the wastewater. The adsorption process of Reactive Black 5 (RB5) by chitosan was studied under batch experimental condition to identify the optimum condition in which the dye can be removed at a higher rate. The best fit kinetics model was determined to be the pseudo-secondorder kinetics. From the isotherm study, the experimental result was better explained by Freundlich isotherm. Plackett-Burman was employed to identify the influential variables affecting the dye uptake. Response surface methodology (RSM) was used to determine the interactions between the factors and their optimum levels for maximum uptake of RB5. The optimum condition for the highest percentage uptake of RB5 dye was determined to be at $\mathrm{pH} 4$, agitation rate of $200 \mathrm{rpm}$, sorbent dosage of $1.0 \mathrm{~g}$, contact time of 300 minutes, and initial dye concentration of $25 \mathrm{mg} / \mathrm{L}$.

\section{Introduction}

Dyes have been used in many different industries to induce colours into different products such as textiles, food, plastics, glass, and more. The worldwide annual production of dyes is about 700 thousand tons, and about $5-15 \%$ of the dyes were discharged into the waste streams by the textile industries alone [1]. Some researchers have also determined that some dyes, dye precursors, and their biotransformation products are toxic, mutagenic, and carcinogenic in nature [2]. Due to these aspects, the dyes discharged into the water streams are actually very dangerous, and might cause health hazards upon consumption. Thus, there has been intensive research in exploring various possibilities to overcome the environmental contamination caused by various dyeing operations.

The commonly used treatment methods for dye removal from aqueous environment include chemical coagulation, activated sludge, trickling filter, carbon adsorption, and photodegradation [3]. Amongst all, the adsorption process can be viewed as one of the most promising methods as there is no byproduct formation; it offers simplicity of the design, and the high abundance of readily available adsorbents makes it an inexpensive method.

Activated carbon can remove organic substances from waste streams at an efficient rate and is most frequently used to remove dyes from waste waters. However, the efficiency of this material is shaded by its expensive treatment cost and the difficulty faced in the regeneration process [4]. Therefore, cheaper naturally available adsorbents were studied to determine their adsorption capacity. Some adsorbents have demonstrated its potential to be low cost adsorbents for dye removal and these include rice husk, orange peel, and sugarcane bagasse [5-7].

Chitosan is an aminopolysaccharide which can be found in the shells of most crustaceans. Chitosan can also be synthesized by deacylation of chitin. Chitin is one of the most 
abundant biopolymers which are naturally biodegradable and nontoxic. The amine functional group on the chitosan is responsible for its acid-base property, solubility, and cationic behaviour. This functional group causes chitosan to be very effective in removing anionic solutes in acidic solutions [8]. Chitosan is also economically cheaper than most adsorbents as it can be obtained from fishery wastes. Therefore, the feasibility of using chitosan as an adsorbent for anionic dye removal was being studied in this research.

Most of the adsorption studies performed through the conventional methods do not or are inadequate to describe the combined effect of all the factors involved. Apart from time consuming, this method also does not guarantee the determination of optimal conditions $[9,10]$. However, these limitations can be overcome by introducing statistical methodology experimental design such as Plackett-Burman and response surface methodology (RSM). Through PlackettBurman, the influential parameters that affect RB5 uptake can be identified, whereas further optimization of the operational condition can be achieved through RSM.

\section{Materials and Methods}

2.1. Preparation of Sorbent. The chitosan used was of analytical grade and in flakes form. The chitosan was made into bead form by dissolving the chitosan flakes into acetic acid. The method used to produce chitosan was extracted from previous studies by other researchers with slight modifications $[11,12]$.

2.2. Preparation of Dye Solutions. Synthetic dye solution of Reactive Black 5 (RB5) was used as the adsorbate in this study. The dye was used without further purification. Standard dye solution of $1000 \mathrm{mg} / \mathrm{L}$ was prepared as stock solution and subsequently diluted when necessary.

2.3. Batch Study. All the batch adsorption experiments were performed in duplicate by agitating a fixed amount of chitosan beads, $0.50 \mathrm{~g}$ in $20 \mathrm{~mL}$ of $25 \mathrm{mg} / \mathrm{L}$ dye solution in a centrifuge tube, and shaken at $150 \mathrm{rpm}$ on an orbital shaker at room temperature $\left(25 \pm 2^{\circ} \mathrm{C}\right)$ unless otherwise stated. The results presented are the means value. Control without sorbent was simultaneously carried out to demonstrate that dye uptake was due to sorbent and not the wall of the centrifuge tube. At the end of the adsorption process, the RB5 dye concentrations were analysed using Perkin Elmer Lambda 35 double-beam UV/visible spectrophotometer with $1.0 \mathrm{~cm}$ light path cuvette (quartz cell) at the wavelength corresponding to maximum absorption, $\lambda_{\max }=654 \mathrm{~nm}$. Dilutions were carried out when the measurement exceeded the linearity of the calibration curve $\left(y=0.0227 ; R^{2}=\right.$ 0.999). Various experimental factors were studied and these included $\mathrm{pH}$, sorbent dosage, concentration and contact time, and agitation rate. For the effect of $\mathrm{pH}$, the study range was between $\mathrm{pH} 3$ and 12 . The effect of initial dye concentrations was studied at 25,50, and $100 \mathrm{mg} / \mathrm{L}$. As for the effect of sorbent dosage, it was varied from $0.1 \mathrm{~g}$ to $1.0 \mathrm{~g}$. The effect of agitation rate was performed by varying the agitation rate from 100 to $150 \mathrm{rpm}$.
2.4. Statistical Analysis via Plackett-Burman and RSM Program. Plackett-Burman design was used to evaluate the relative importance of the various factors that influence the percentage uptake of the system. The purpose of applying this design is to identify the significant factor(s) that will affect the percentage uptake of RB5. In this study, there are 4 assigned variables (concentration and contact times, sorbent dosage, agitation rate, and $\mathrm{pH}$ ) which were screened in 12 experimental designs. The RSM approach uses the central composite design (CCD) model to study the variables used for the studied dye at 3 coded levels $(-1,0$, and +1$)$ for RB5 dye. The cubic equation was used for the optimization of the percentage of dye, which is shown as

$$
\begin{aligned}
Y= & \beta_{o}+\sum_{i=1}^{2} \beta_{i} X_{i}+\sum_{i=1}^{2} \beta_{i i} X_{i}^{2} \\
& +\sum_{i=1}^{2} \beta_{i i i} X_{i}^{3}+\sum_{i=1}^{1} \sum_{j=i+1}^{2} \beta_{i j} X_{i} X_{j} \\
& +\sum_{i=1}^{1} \sum_{j=i+1}^{2} \beta_{i j j} X_{i} X_{j}^{2} \\
& +\sum_{i=1}^{1} \sum_{j=i+1}^{2} \beta_{i i j} X_{i}^{2}+X_{j},
\end{aligned}
$$

where $\beta_{o}, \beta_{i}, \beta_{i i}, \beta_{i i i}, \beta_{i j}, \beta_{i j j}$, and $\beta_{i i j}$ are the constant coefficients and $X_{i}$, and $X_{j}$ are the independent variables. All experimental designs and statistical analyses of the data were done by using Design Expert Version 7.1.3. All experiments were conducted in duplicate and the mean value of the duplicates was taken as the response (percentage uptake of dyes).

\section{Results and Discussion}

3.1. Results of Batch Study. From the batch study, it was determined that chitosan adsorbs best under the influence of low $\mathrm{pH}$, high sorbent dosage, and low initial dye concentration. The agitation rate effect on $\mathrm{RB} 5$ uptake is of minimal. The $\mathrm{pH}$ affects the percentage uptake of RB5 dye by protonating the amine functional group of chitosan, thus, making the binding sites available for anionic dye. In the $\mathrm{pH}$ range of 4 to 6 , an appreciable amount of RB5 could be sorbed by chitosan. As this is also the range of natural pH of RB5 dye solution, thus, no $\mathrm{pH}$ adjustment was carried out for the further parameters studied. The increase in sorbent dosage leads to a higher percentage uptake of dye by chitosan because with increasing amount of adsorbents, the availability of active binding sites increases proportionally. With increasing dye concentration, a lower uptake of RB5 was recorded, and this is due to higher dye concentration causing the binding sites to be saturated with dye molecules at a faster rate. When the available active sites are saturated with dye molecules, effective adsorption will be restricted, and therefore, a lower uptake is anticipated. The various operational parameters in the aforementioned section were further studied under statistical approach to 


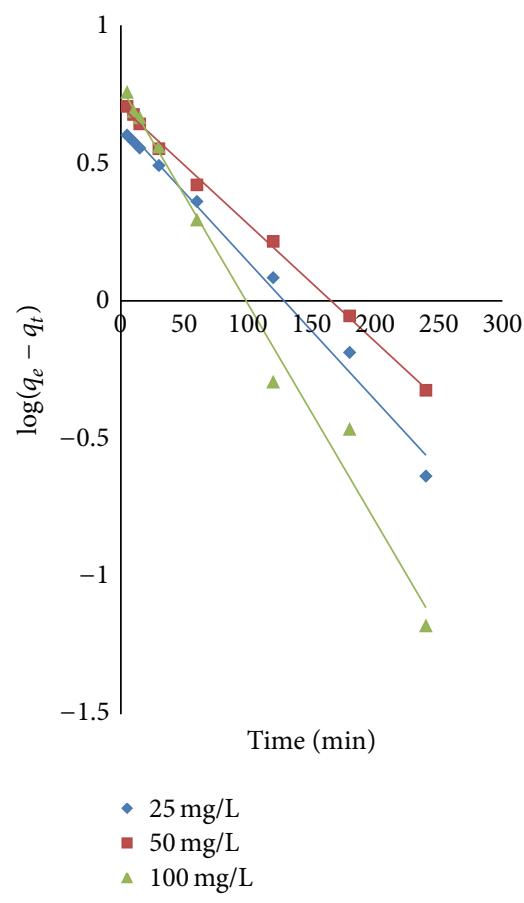

Figure 1: Pseudo-first-order graph for the effect of concentration and contact time.

identify the influential factors and to determine the optimum condition for RB5 uptake by chitosan.

3.2. Kinetic Studies. From the results obtained, the pseudofirst- and pseudo-second-order kinetic graphs were plotted by applying the pseudo-first-order kinetic rate equation [13] and pseudo-second-order kinetic rate equation [14] which can be expressed as

$$
\begin{aligned}
\log \left(q_{e}\right. & \left.-q_{t}\right) \\
& =\log \left(q_{e}\right)-\frac{k_{1} t}{2.303} \quad \text { (pseudo-first order equation), } \\
\frac{t}{q_{t}} & =\frac{1}{h}+\frac{t}{q_{e}} \quad \text { (pseudo-second order equation), }
\end{aligned}
$$

where $q_{e}=$ the amount of RB5 adsorbed at equilibrium $(\mathrm{mg} / \mathrm{g}), q_{t}=$ the amount of RB5 adsorbed at time $t(\mathrm{mg} / \mathrm{g})$, $k_{1}=$ the rate constant of pseudo-first-order kinetics $(1 / \mathrm{min})$, $h\left(k_{2} q_{e}^{2}\right)=$ the initial adsorption rate ( $\left.\mathrm{mg} / \mathrm{g} \min \right)$, and $k_{2}=$ the rate constant of pseudo-second-order kinetics (g/mg min).

From Figures 1 and 2, based on the correlation coefficient value, $R^{2}$ (Table 1), it indicates that the system under study is more appropriately described by the pseudo-second-order model which was based on the assumption that the rate limiting step may be chemical adsorption or chemisorption involving valency forces through sharing or exchange of electron between adsorbent and adsorbate [15]. Besides, the equilibrium adsorption capacities determined from the first-order kinetic model were lower than those determined experimentally. It thus appears that the system under study

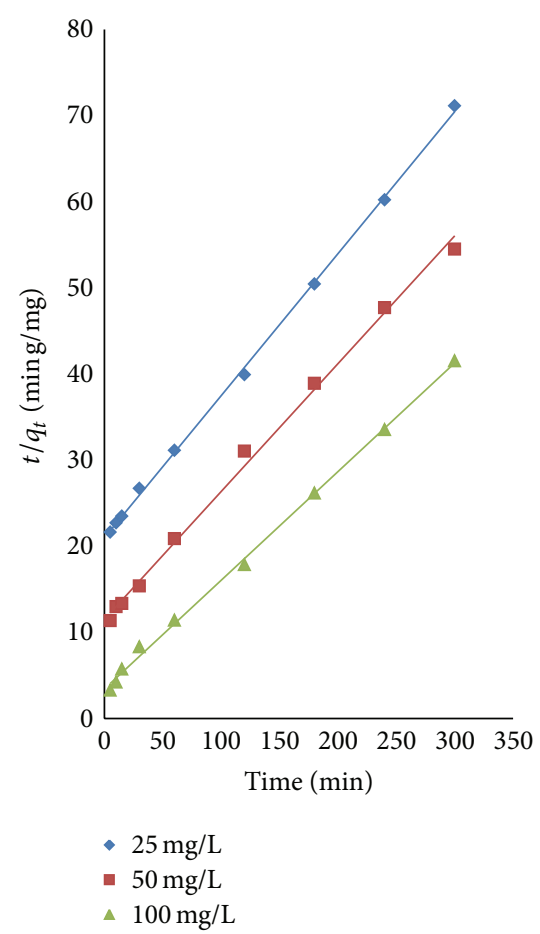

Figure 2: Pseudo-second-order graph for the effect of concentration and contact times.

is more appropriately described by the pseudo-second-order model. The applicability of pseudo-second-order kinetics in the adsorption process of various biosorbents has also been well documented [15-17].

From the results obtained, the values of $q_{e}, k_{2}$, and $h$ were plotted against $C_{o}$ to obtain a corresponding graph that shows the relationship between the theoretical values and the experimental values. These values can be expressed with the following equations:

$$
\begin{aligned}
& q_{e}=\frac{C_{o}}{A_{q} C_{o}+B_{q}} \\
& k_{2}=\frac{C_{o}}{A_{k} C_{o}+B_{k}} \\
& h=\frac{C_{o}}{A_{h} C_{o}+B_{h}},
\end{aligned}
$$

where $A_{q}, B_{q}, A_{k}, B_{k}, A_{h}$, and $B_{h}$ are constants. The constant values can then be determined from the slopes, and intercepts of the linear plots and generate the predictive model for RB5 uptake at any contact time and initial concentrations within the given range. The theoretical model for RB5 uptake can be represented by the following equation:

$$
q_{t}=\frac{C_{o} t}{-1.3609 C_{o}+582.52+\left(0.1179 C_{o}+1.2471\right) t} .
$$

Figure 3 shows the pseudo-second-order modelled time profile for RB5 adsorption by chitosan beads. From the graph, it is evident that the experimental values lay closely 
TABLE 1: Comparison between pseudo-first-order kinetic model and pseudo-second-order kinetic model.

\begin{tabular}{|c|c|c|c|c|c|c|c|c|}
\hline \multirow{2}{*}{$\begin{array}{l}\text { Initial RB5 } \\
\text { concentration (mg/L) }\end{array}$} & \multirow{2}{*}{$q_{e, \exp }(\mathrm{mg} / \mathrm{g})$} & \multicolumn{3}{|c|}{ Pseudo-first-order kinetic model } & \multicolumn{4}{|c|}{ Pseudo-second-order kinetic model } \\
\hline & & $q_{e, \mathrm{cal}}(\mathrm{mg} / \mathrm{g})$ & $k_{1}\left(\times 10^{-3} 1 / \mathrm{min}\right)$ & $R^{2}$ & $q_{e, \mathrm{cal}}(\mathrm{mg} / \mathrm{g})$ & $k_{2}\left(\times 10^{-3} \mathrm{~g} / \mathrm{mg} \min \right)$ & $h(\mathrm{mg} / \mathrm{g} \min )$ & $R^{2}$ \\
\hline 25 & 6.22 & 4.41 & 11.52 & 0.990 & 6.07 & 1.29 & 0.05 & 0.999 \\
\hline 50 & 6.51 & 5.07 & 9.90 & 0.997 & 6.74 & 1.91 & 0.09 & 0.996 \\
\hline 100 & 7.22 & 5.97 & 18.19 & 0.985 & 7.92 & 4.65 & 0.30 & 0.998 \\
\hline
\end{tabular}

TABLE 2: Comparison of Langmuir isotherm with Freundlich isotherm.

\begin{tabular}{lcccccrr}
\hline \multirow{2}{*}{ Sorbent } & \multirow{2}{*}{ Dye } & Langmuir & \multicolumn{3}{c}{ Freundlich } \\
& & $N^{*}(\mathrm{mg} / \mathrm{g})$ & $b(1 / \mathrm{mg})$ & $R^{2}$ & $K_{f}$ & $R^{2}$ \\
\hline Chitosan & RB5 & 8.14 & 0.10 & 0.992 & 2.43 & 3.99 \\
\hline
\end{tabular}

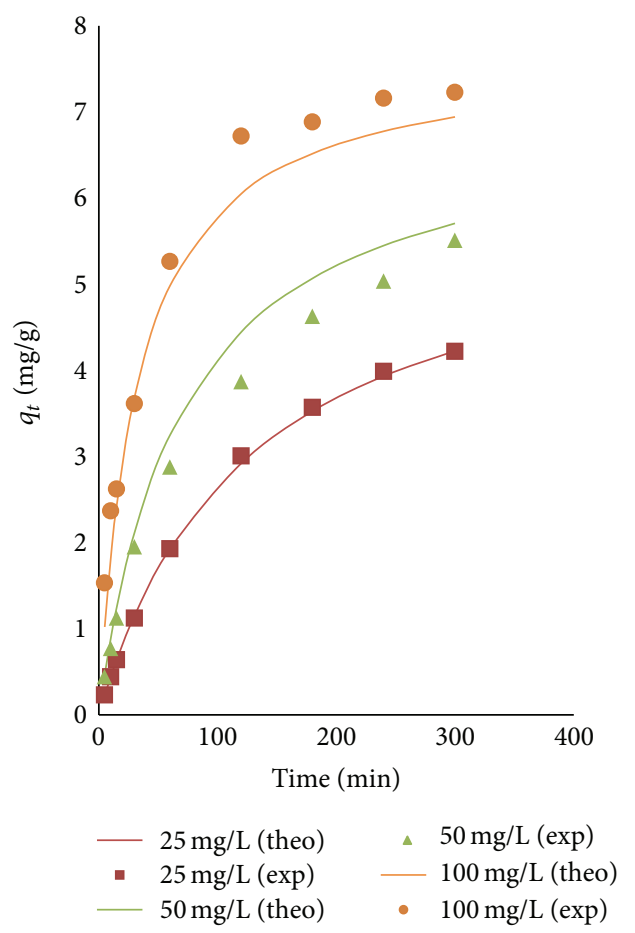

FIGURE 3: Comparison between the theoretical and experimental $q_{t}$ values generated by the theoretical model for RB5.

to the theoretical values regardless of their concentrations. This again indicates the applicability and reliability of the pseudo-second-order kinetic model in describing the current adsorption process.

3.3. Langmuir Isotherm. The equilibrium adsorption data of RB5 on chitosan was fitted into Langmuir isotherm equation, which featured four main assumptions [18]:

(a) the adsorption process only takes place at specific sites on the surface and the saturation coverage corresponds to the maximum occupancy of the sites;

(b) each site can only accommodate one molecule or atoms; (c) the surface of the adsorbent is energetically homogenous hence there is no interaction between the adjacent adsorbed molecules;

(d) there are no phase transitions.

The equation of the linearised Langmuir model can be written as

$$
\frac{C_{e}}{N_{e}}=\frac{1}{N^{*} b}+\frac{C_{e}}{N^{*}}
$$

where $C_{e}=$ equilibrium concentration of dye $(\mathrm{mg} / \mathrm{L}), N_{e}=$ amount of dye adsorbed at equilibrium (mg/g), $N^{*}=$ maximum sorption capacity $(\mathrm{mg} / \mathrm{g})$, and $b=$ constant which is related to the energy of the sorbent $(\mathrm{L} / \mathrm{mg})$.

The coefficients of the linearised form of Langmuir isotherm model are tabulated in Table 2.

Besides, the Langmuir isotherm could also be expressed in a dimensionless constant separation factor $\left(R_{L}\right)$ to indicate the isotherm shape [19]. Based on the shape of the isotherm, the adsorption system can be predicted as favourable or unfavourable,

$$
R_{L}=\frac{1}{1+b C_{o}}
$$

where $C_{o}=$ initial concentration of dye $(\mathrm{mg} / \mathrm{L})$ and $b=$ constant which is related to the energy of the sorbent $(\mathrm{L} / \mathrm{mg})$.

The $R_{L}$ values for each of the concentrations were calculated, and based on the results obtained, it showed that the adsorption system in this study corresponds to a favourable adsorption process. The value of $R_{L}$ decreases from 0.286 to 0.091 as the concentration increases from $25 \mathrm{mg} / \mathrm{L}$ to $100 \mathrm{mg} / \mathrm{L}$. This decrease actually indicates that as the concentration is increased, the isotherm tends to skew towards the irreversible case.

3.4. Freundlich Isotherm. The Freundlich isotherm is used to describe the adsorption over a wider range of dye concentrations and it is accounted for nonlinear adsorption behaviour. The assumption of the Freundlich isotherm is that the uptake of dye molecules occurred on a heterogeneous surface by which multilayer adsorption and the concentration of adsorbates on the surface will increase as concentration 
TABLE 3: Plackett-Burman design and results for the uptake of RB5 by chitosan beads.

\begin{tabular}{lcccccc}
\hline No. & Agitation rate $(\mathrm{rpm})$ & Chitosan dosage $(\mathrm{g})$ & Concentration $(\mathrm{mg} / \mathrm{L})$ & $\mathrm{pH}$ & Predicted response $(\%)$ & Observed response $(\%)$ \\
\hline 1 & 100.00 & 0.10 & 25.00 & 12.00 & 7.16 & 8.95 \\
2 & 200.00 & 1.00 & 25.00 & 12.00 & 26.99 & 31.27 \\
3 & 100.00 & 1.00 & 100.00 & 12.00 & 6.91 & 5.13 \\
4 & 197.32 & 0.74 & 70.75 & 11.89 & 13.24 & 9.52 \\
5 & 103.02 & 0.20 & 40.42 & 11.95 & 6.24 & 10.02 \\
6 & 125.42 & 0.12 & 43.26 & 3.25 & 84.04 & 89.32 \\
\hline
\end{tabular}

TABLE 4: Regression analysis (ANOVA) generated by the Plackett-Burman.

\begin{tabular}{|c|c|c|c|c|c|}
\hline Source & Sum of squares & Degree of freedom & Mean square & $F$ value & $\begin{array}{c}P \text { value } \\
\text { Prob }>F\end{array}$ \\
\hline Model & 18751.7745 & 4 & 4687.9436 & 40.0002 & $<0.0001$ \\
\hline A-Agitation rate & 41.0410 & 1 & 41.0410 & 0.3502 & 0.5726 \\
\hline$B$-Chitosan dosage & 751.1794 & 1 & 751.1794 & 6.4095 & 0.0391 \\
\hline C-Concentration & 774.8643 & 1 & 774.8643 & 6.6116 & 0.0369 \\
\hline$D-\mathrm{pH}$ & 17806.1833 & 1 & 17806.1833 & 151.9326 & $<0.0001$ \\
\hline
\end{tabular}

of adsorbates in the liquid phase increases. This isotherm is much more flexible as compared to the Langmuir and the Freundlich isotherm does not indicate adsorption limit.

The linear form of the Freundlich can be written as

$$
\log N_{e}=\frac{\log C_{e}}{n}+\log K_{f}
$$

where $C_{e}=$ equilibrium concentration of dye $(\mathrm{mg} / \mathrm{L}), N_{e}=$ amount of dye adsorbed at equilibrium (mg/g), $n=$ Freundlich constant for intensity, and $K_{f}=$ Freundlich constant for sorption capacity ( $\mathrm{mg} / \mathrm{g})$.

The coefficients of the Freundlich isotherm are calculated and presented in Table 2 . The Freundlich adsorption capacity $K_{f}$, isotherm intensity $n$, and correlation coefficient $R^{2}$ were $2.43 \mathrm{mg} / \mathrm{g}, 3.99$, and 0.994 , respectively. From the value of $n$ which is 3.99 , it indicates that the magnitude of favourability of the adsorption is within the range of 1-10, thus, revealing a favourable adsorption [20]. From the results obtained, the value of $n$ is greater than 1 which also indicates that the extent of adsorption increases with increasing dye concentration [21].

The high correlation coefficients, $R^{2}$, showed that both isotherm models were capable to provide reasonable fittings for the adsorption data. Although these two models are based on different postulations, fitting the model to the sorption process does not necessarily imply any physical interpretation attached to them since the biosorbent's surface is nonhomogeneous and there could be more than one type of sorption sites on the biosorbent's surface [22].

3.5. Statistical Approach (Plackett-Burman and RSM). In order to overcome the limitations found in conventional and classical methods of studying a process, the current study incorporates the statistical experimental methodology to identify and determine the influential factors for further optimization. Table 3 shows the Plackett-Burman design and the results obtained from the experimental runs. In this study, 4 assigned variables, namely, $\mathrm{pH}$, initial dye concentration, chitosan dosage, and agitation rate, were screened in 12 experimental designs, and Table 3 shows some of the selected results from the experimental designs. It is evident that from all the conducted experiments, the results obtained in terms of their percentage uptake were very close as compared to those estimated values generated by the program. Therefore, it can be concluded that the results obtained via the program are very accurate, thus, this program is usable to study the different effects of parameters on the percentage uptake of dye by chitosan.

From the analysis of variance (ANOVA), the assigned variable is considered as a significant factor when the Prob $>F$ was less than 0.05 . Therefore, it can be concluded that 3 out of 4 assigned variables are actually significant in this study (Table 4). These include $\mathrm{pH}$, initial dye concentration, and chitosan dosage. From the same table, Prob $>F$ is having the value $<0.0001$, which indicates that this model was significant.

The $\mathrm{pH}$ parameter is known to be one of the most influential parameters in adsorption process because it affects the surface charge of the adsorbent and plays an important role in the degree of ionization. The functional groups that was responsible for the dye uptake is affected by the $\mathrm{pH}$ of the dye solution. The result from this study showed that the efficient uptake occurred in acidic medium. Apart from this factor, the initial dye concentration and the amount of chitosan also played a significant role in affecting the RB5 uptake. The uptake of RB5 decreases with increasing dye concentrations whereas a higher dosage of chitosan facilitates a better uptake and this corresponds to an increase in active sites for sorption.

The central composite design (CCD) model was used in this study. The variables used for the studied dye at 3 coded levels $(-1,0$, and +1$)$ for RB5 are shown in Table 5 . From the CCD model, a few designed matrixes of independent variable 
TABLE 5: Experimental range and levels of independent variables of RB5.

\begin{tabular}{|c|c|c|c|c|c|}
\hline \multirow{2}{*}{ Dye } & \multirow{2}{*}{ Factors } & \multirow{2}{*}{ Factor code } & \multicolumn{3}{|c|}{ Range and levels (coded) } \\
\hline & & & -1 & 0 & +1 \\
\hline \multirow{3}{*}{ RB5 } & $\mathrm{pH}$ & $A$ & 3.00 & 7.50 & 12.00 \\
\hline & Sorbent dosage (g) & $B$ & 0.10 & 0.55 & 1.00 \\
\hline & Initial dye concentration $(\mathrm{mg} / \mathrm{L})$ & $C$ & 25.00 & 62.50 & 100.00 \\
\hline
\end{tabular}

TABLE 6: CCD matrix for 3 independent variables and observed response for RB5.

\begin{tabular}{lccccc}
\hline Experimental run & $A$ & $B$ & $C$ & Observed response (\%) & Predicted response (\%) \\
\hline 1 & +1 & +1 & +1 & 7.63 & 11.01 \\
2 & +1 & -1 & -1 & 8.94 & 12.42 \\
3 & +1 & +1 & -1 & 31.27 & 28.07 \\
4 & -1 & +1 & 0 & 99.95 & 99.82 \\
5 & -1 & 0 & -1 & 99.79 & 97.88 \\
\hline
\end{tabular}

TABLE 7: Regression analysis (ANOVA) for the uptake of RB5.

\begin{tabular}{lcccr}
\hline Source & Sum of square & Degree of freedom & Mean square & $F$ value \\
\hline Model & 13408.46 & 3 & 4469.49 & 47.58 \\
$A$ & 489.79 & 1 & 489.79 & 5.21 \\
$B$ & 581.91 & 1 & 581.91 & 6.0014 \\
$C$ & 12336.76 & 1 & 12336.76 & 0.0845 \\
Residual & 375.75 & 4 & 93.94 & 0.0676 \\
\hline
\end{tabular}

$R^{2}: 0.973$, adjusted $R^{2}: 0.952$, predicted $R^{2}: 0.891$, adequate precision: 16.23 , and C.V.: 19.01 .

with their respective responses were chosen and are shown in Table 6. The experiments were conducted twice to obtain a mean value for the results.

From the RSM results, RB5 dye solution shows a percentage uptake rate that follows the following equation which was generated by the Design-Expert 7.1.3:

$$
\begin{aligned}
\% \text { uptake }= & 121.0827111 \\
& +17.38797222 \times B-0.227432333 \\
& \times C-8.726558333 \times A,
\end{aligned}
$$

where $A=\mathrm{pH}, B=$ sorbent dosage, and $C=$ concentration of dye.

The designed model is termed as significant when the Prob $>F$ value is lower than 0.05. Therefore, from the ANOVA analysis, it was determined that the model is significant as the Prob $>F$ value is 0.0014 (Table 7). The $R^{2}$ value for RB5 was reported to be 0.973 . This value is relatively high as it is close to unity, and therefore, it is estimated that there will be good agreement between the experimental and predicted results.

By using the surface plot, the interactions between the two factors and their optimum levels can be studied. From the 3D plot, the maximum uptake should occur when sorbent dosage was at maximum, whereas the initial dye concentration was at minimum. In order to verify the data generated from the $3 \mathrm{D}$ plot, experiments were conducted to verify the value predicted from the program. The theoretical maximum uptake is $97.88 \%$, and through the experiment, the uptake was
99.79\% (under the condition: $\mathrm{pH} 4.0$, sorbent dosage $1.0 \mathrm{~g}$, and initial dye concentration $25 \mathrm{mg} / \mathrm{L}$ ). The percentage error of the result is $1.91 \%$. In conclusion, the model generated by the program Design-Expert Version 7.1.3 is valid [23].

\section{Conclusion}

Chitosan beads can serve as a potential adsorbent for the removal of RB5 dye from aqueous solution. The equilibrium data conform to both Langmuir and Freundlich isotherms with the maximum sorption capacity of $8.14 \mathrm{mg} / \mathrm{g}$ for RB5. For the system under examination, pseudo-second-order kinetic model provided a better correlation of the experimental data than pseudo-first-order equation of Lagergren model. From the statistical study, the results revealed that in acidic medium, low initial dye concentration and high chitosan dosage will lead to a better dye uptake. Under the optimum operating condition, the predicted RB5 uptake can be greater than $99 \%$, and this value agreed well with the experimental results.

\section{Acknowledgments}

This work received financial support from the International Foundation of Science, Stockholm, Sweden, and the Organisation for the Prohibition of Chemical Weapons, The Hague, Netherlands, via Grant no. W/4368-1, and the laboratory facilities provided by Universiti Tunku Abdul Rahman (UTAR) are acknowledged. 


\section{References}

[1] J.-W. Lee, S.-P. Choi, R. Thiruvenkatachari, W.-G. Shim, and H. Moon, "Evaluation of the performance of adsorption and coagulation processes for the maximum removal of reactive dyes," Dyes and Pigments, vol. 69, no. 3, pp. 196-203, 2006.

[2] W. H. Cheung, Y. S. Szeto, and G. McKay, "Enhancing the adsorption capacities of acid dyes by chitosan nano particles," Bioresource Technology, vol. 100, no. 3, pp. 1143-1148, 2009.

[3] S. Chatterjee, S. Chatterjee, B. P. Chatterjee, A. R. Das, and A. K. Guha, "Adsorption of a model anionic dye, eosin Y, from aqueous solution by chitosan hydrobeads," Journal of Colloid and Interface Science, vol. 288, no. 1, pp. 30-35, 2005.

[4] F. Banat, S. Al-Asheh, R. Al-Ahmad, and F. Bni-Khalid, "Benchscale and packed bed sorption of methylene blue using treated olive pomace and charcoal," Bioresource Technology, vol. 98, no. 16, pp. 3017-3025, 2007.

[5] A. E. Nemr, O. Abdelwahab, A. El-Sikaily, and A. Khaled, "Removal of direct blue- 86 from aqueous solution by new activated carbon developed from orange peel," Journal of Hazardous Materials, vol. 161, no. 1, pp. 102-110, 2009.

[6] S.-T. Ong, E.-C. Khoo, P.-S. Keng et al., "Plackett-Burman design and response surface methodological approach to optimize basic dyes removal using sugarcane bagasse," Desalination and Water Treatment, vol. 25, no. 1-3, pp. 310-318, 2011.

[7] R. Han, D. Ding, Y. Xu et al., "Use of rice husk for the adsorption of congo red from aqueous solution in column mode," Bioresource Technology, vol. 99, no. 8, pp. 2938-2946, 2008.

[8] E. Guibal and J. Roussy, "Coagulation and flocculation of dyecontaining solutions using a biopolymer (Chitosan)," Reactive and Functional Polymers, vol. 67, no. 1, pp. 33-42, 2007.

[9] A. Rajendran, M. Thirugnanam, and V. Thangavelu, "Statistical evaluation of medium components by Plackett-Burman experimental design and kinetic modeling of lipase production by Pseudomonas fluorescens," Indian Journal of Biotechnology, vol. 6, no. 4, pp. 469-478, 2007.

[10] U. K. Garg, M. P. Kaur, V. K. Garg, and D. Sud, "Removal of Nickel(II) from aqueous solution by adsorption on agricultural waste biomass using a response surface methodological approach," Bioresource Technology, vol. 99, no. 5, pp. 1325-1331, 2008.

[11] D. Kołodyńska, "Adsorption characteristics of chitosan modified by chelating agents of a new generation," Chemical Engineering Journal, vol. 179, pp. 33-43, 2012.

[12] S. Y. Jeong, I. C. Kwon, K. Choi, and E. Bae, "Macroporous chitosan beads and preparation method thereof," Patent Application Publication, pp. 1-3, 2007.

[13] S. Langergren and B. K. Svenska, "Zur theorie der sogenannten adsorption geloester stoffe," Veternskapsakad Handlingar, vol. 24, pp. 1-39, 1898.

[14] Y. S. Ho and G. McKay, "Pseudo-second order model for sorption processes," Process Biochemistry, vol. 34, no. 5, pp. 451$465,1999$.

[15] Y. S. Ho and G. McKay, "The kinetics of sorption of divalent metal ions onto sphagnum moss peat," Water Research, vol. 34, no. 3, pp. 735-742, 2000.

[16] S. T. Ong, S. T. Ha, E. C. Khoo, and S. L. Hii, "Nitrilotriacetic acid modified sugarcane bagasee in the removal of basic blue 3 from aqueous environment," International Journal of Environmental Engineering, vol. 5, pp. 299-310, 2013.
[17] S. T. Ong, S. Y. Tan, E. C. Khoo, S. L. Lee, and S. T. Ha, "Equlibrium studies for Basic blue 3 adsorption onto durain peel *Durio zibethinus Murray," Desalination and Water Treatment Journal, vol. 45, pp. 161-169, 2012.

[18] C. Leszek, M. Balys, and K. Ewa, "Some generalization of Langmuir adsorption isotherm," Internet Journal of Chemistry, vol. 3, pp. 1099-8292, 2000.

[19] K. Kadirvelu, K. Thamaraiselvi, and C. Namasivayam, "Removal of heavy metals from industrial wastewaters by adsorption onto activated carbon prepared from an agricultural solid waste," Bioresource Technology, vol. 76, no. 1, pp. 63-65, 2001.

[20] R. Sivaraj, C. Namasivayam, and K. Kadirvelu, "Orange peel as an adsorbent in the removal of Acid violet 17 (acid dye) from aqueous solutions," Waste Management, vol. 21, no. 1, pp. 105110, 2001.

[21] T. K. Saha, N. C. Bhoumik, S. Karmaker, M. G. Ahmed, H. Ichikawa, and Y. Fukumori, "Adsorption of methyl orange onto chitosan from aqueous solution," Journal of Water Resource and Protection, vol. 2, pp. 898-906, 2010.

[22] B. Volesky, "Biosorption and biosorbents," in Biosorption of Heavy Metals, B. Volesky, Ed., pp. 3-44, CRC Press, Fla, USA, 1990.

[23] J.-K. Wang and C.-S. Hsieh, "Determination of optimal conditions for separation of metal ions through membrane dialysis using statistical experimental methods," Desalination and Water Treatment, vol. 2, no. 1-3, pp. 240-247, 2009. 

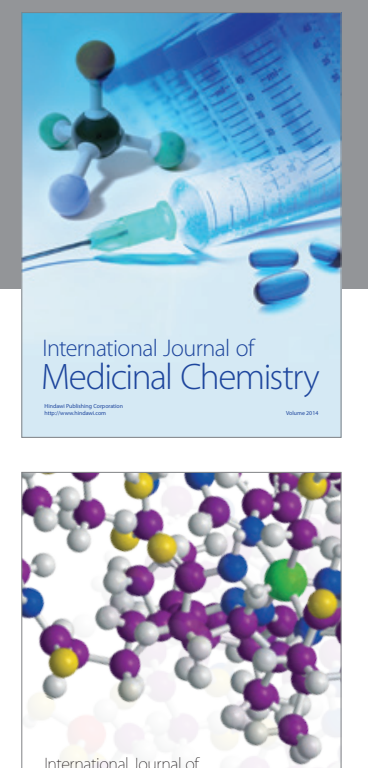

\section{Carbohydrate} Chemistry

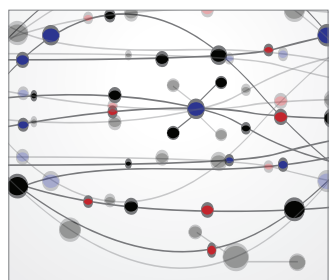

The Scientific World Journal
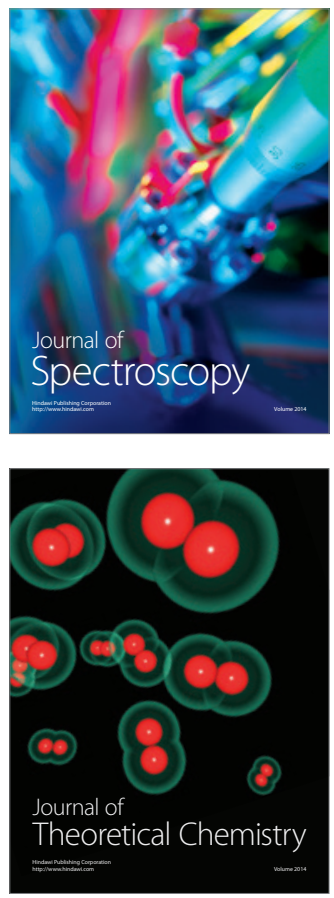
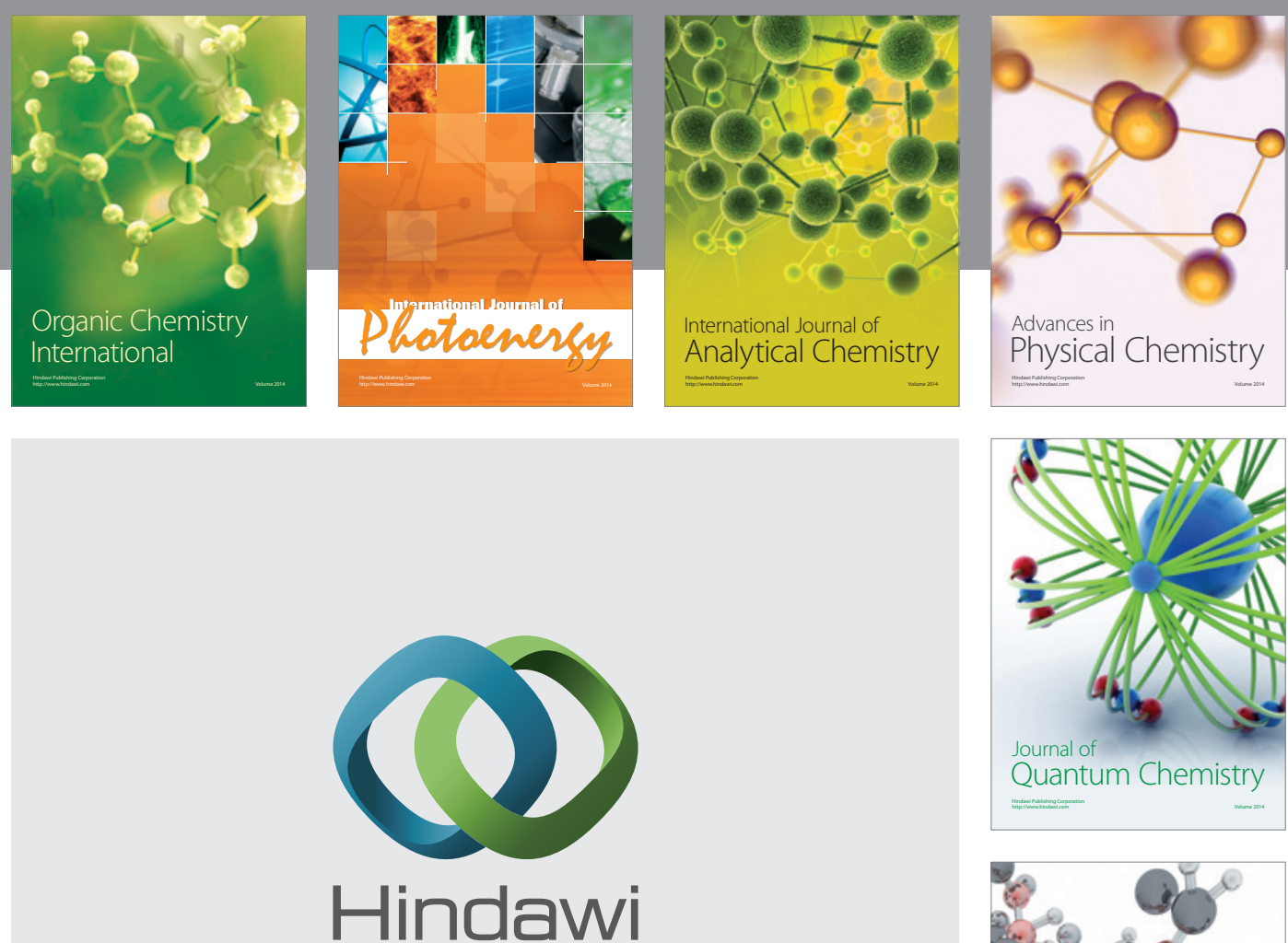

Submit your manuscripts at

http://www.hindawi.com

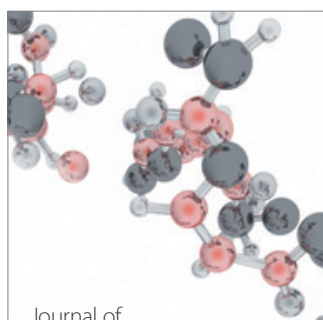

Analytical Methods

in Chemistry

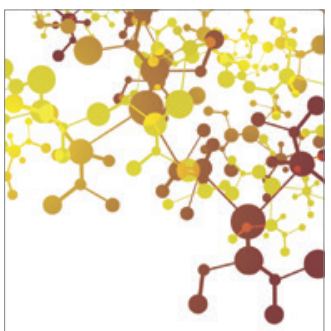

Journal of

Applied Chemistry

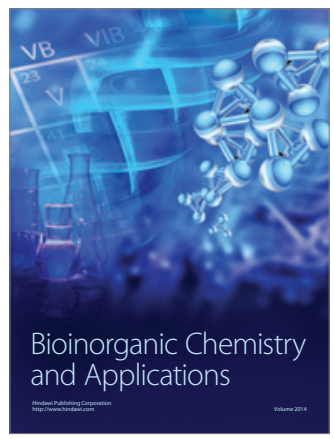

Inorganic Chemistry
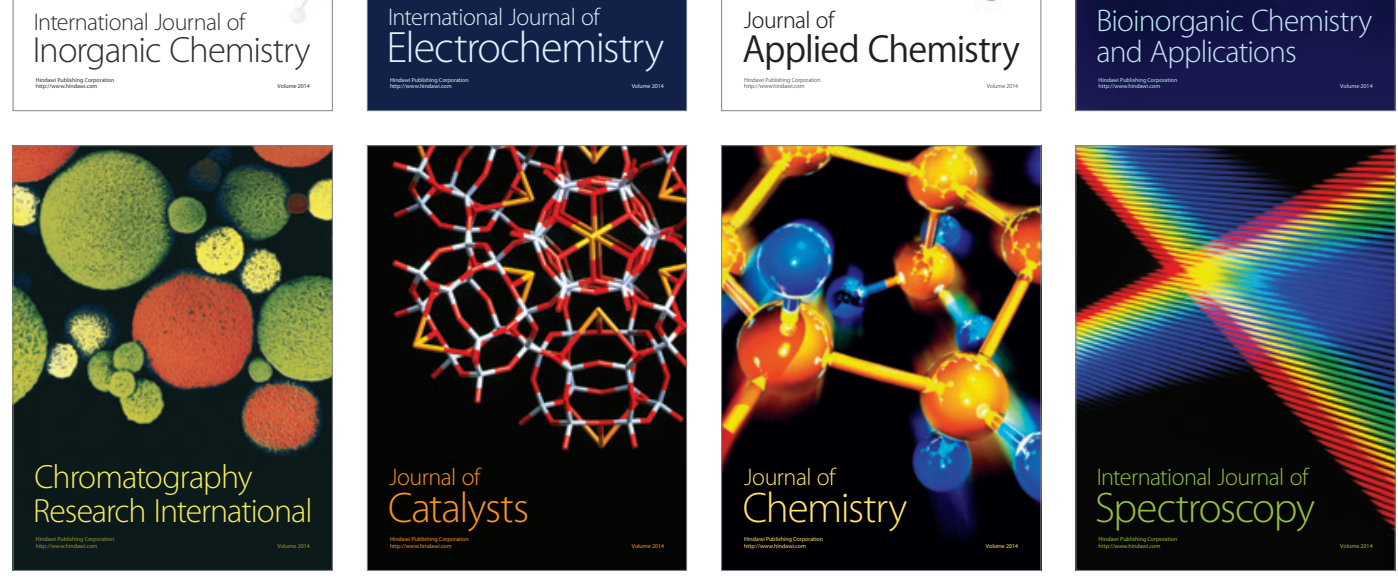